\title{
Is There a Gender Difference in Fat Distribution around the Hamstring Tendon Insertion? A Prospective MRI Evaluation of 40 Cases
}

\author{
Nathanael Ahearn, Nick R. Howells, and James L. Williams \\ Department of Trauma and Orthopaedic Surgery, Musgrove Park Hospital, Parkfield Drive, Taunton, Somerset TA1 5DA, UK \\ Correspondence should be addressed to Nathanael Ahearn; nahearn@nhs.net
}

Received 4 June 2014; Revised 27 July 2014; Accepted 29 July 2014; Published 11 August 2014

Academic Editor: Federico Canavese

Copyright (C) 2014 Nathanael Ahearn et al. This is an open access article distributed under the Creative Commons Attribution License, which permits unrestricted use, distribution, and reproduction in any medium, provided the original work is properly cited.

Introduction. Knee ligament reconstructions are commonly performed using hamstring tendon grafts. We observed anecdotally that there was a difference in the fat distribution superficial to the pes anserinus between men and women and proposed that this effect was independent of BMI, being significantly greater in women. Methods. We performed a prospective study to evaluate 40 MRI scans performed in 20 women and 20 men. The scans allowed visualisation of the insertion of the hamstring tendons at the pes anserinus. Results. The mean BMI of the male patients was $25.6(19.8-37.2)$ and of the female patients was 24.7 (17.9-34.5). The mean fat distribution superficial to the pes anserinus in men was $16.2 \mathrm{~mm}(4.1-29.4)$ and in women was $29.7 \mathrm{~mm}(19.6-47.5)$. There was a significant increase in fat superficial to the hamstring tendons in women compared with men $(P<0.001)$, despite no significant difference in BMI $(P=0.5)$. Conclusions. Our evaluation of a prospective series of MRI scans has shown that there is significantly more fat superficial to the insertion of the hamstring tendons in women than in men. This effect is independent of BMI and may influence exposure during hamstring tendon graft harvesting.

\section{Introduction}

Knee ligament reconstructions are commonly performed using hamstring tendon grafts. Whilst performing hamstring tendon graft harvesting for knee ligament reconstructions, we observed that there was a difference in the fat distribution superficial to the pes anserinus between men and women. We felt that this effect was independent of body mass index (BMI), being significantly greater in women. Women and men of similar BMI differ in the percentage of body weight as fat [1], but it has not been determined if this is related specifically to lower limb anatomy. There have been studies demonstrating gender differences of various aspects of knee anatomy and function such as medial femoral condylar height [2] and rotational kinematics and trochlear groove orientation [3]. To our knowledge there have been no studies investigating gender differences regarding the anatomy of the region around the hamstring tendons.
We therefore designed a prospective study to evaluate a series of magnetic resonance imaging (MRI) scans to determine if there was a difference in the fat superficial to the pes anserinus.

\section{Patients and Methods}

The study used a total of 40 MRI scans, 20 scans performed in men and 20 scans performed in women. Patients under 16 years of age were excluded from the study. We used a consecutive series of scans that were performed for assessment of patellar instability. These scans allowed us to identify the insertion of the pes anserinus on the T1-weighted axial images. We used the Picture Archiving and Communications System (PACS) Centricity Web software (GE Healthcare, Chalfont St Giles, UK) to measure the perpendicular distance from the pes anserinus to the skin. The patient's height and weight information used to calculate BMI were recorded on a 


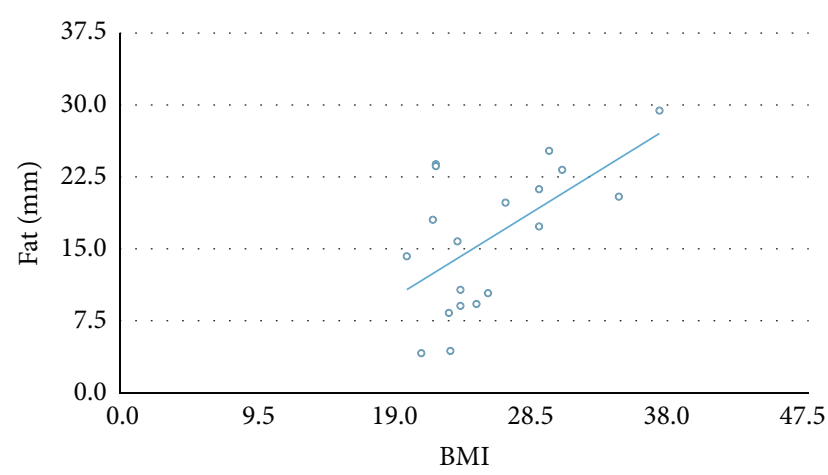

(a) Male fat versus BMI

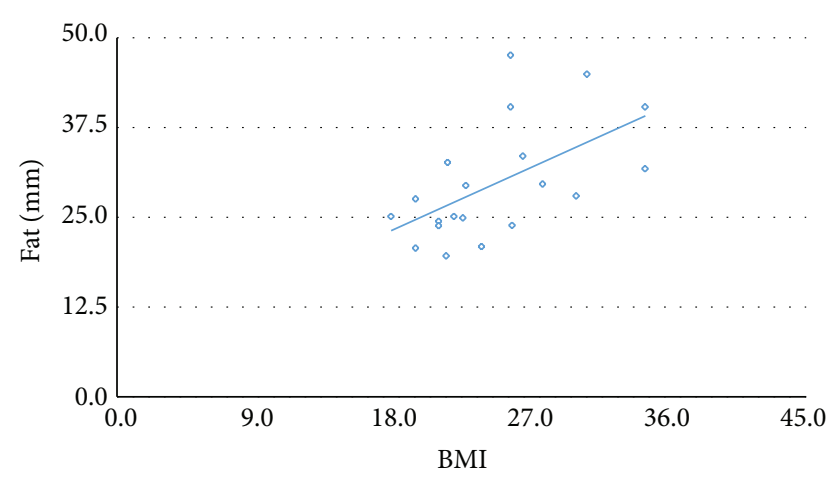

(b) Female fat versus BMI

FIGURE 1: Correlation of the fat distribution and the BMI in male and female patients.

TABLE 1: Summary of the characteristics of male and female patients.

\begin{tabular}{lccc}
\hline & Male & Female & $P$ value \\
\hline $\begin{array}{l}\text { Knee involved } \\
\text { \% right })\end{array}$ & 55 & 55 & - \\
$\begin{array}{l}\text { Age (years) } \\
\text { BMI }\end{array}$ & $29(17-48)$ & $28(17-47)$ & 0.5 \\
$\begin{array}{l}\text { Fat superficial to } \\
\text { pes anserinus }\end{array}$ & $16.2 \mathrm{~mm}(4.1-29.4)$ & $29.7 \mathrm{~mm}(19.6-47.5)$ & $<0.001$ \\
\hline
\end{tabular}

database used for a previous study [4] and correlated with the MRI measurements. All patients had an ethnic origin defined as white British or European.

\section{Statistical Analysis}

A prospective power analysis was conducted to determine the required number of MRI scans needed to evaluate the difference in fat superficial to the pes anserinus. A minimal sample size of 16 in each group would provide $80 \%$ power to detect a difference in means of $5 \mathrm{~mm}$ assuming a common standard deviation (SD) of $8 \mathrm{~mm}$ and $5 \%$ significance. A two-tailed Student's $t$-test was used to determine statistical significance taken at a $P$ value $<0.05$.

\section{Results}

The mean age of the male patients was 29 years (17-48) and that of the female patients was 28 years (19-47). There were a total of 22 right knees and 18 left knees. The mean BMI of the male patients was $25.6(19.8-37.2)$ and that of the female patients was 24.7 (17.9-34.5). The mean fat distribution superficial to the pes anserinus in men was $16.2 \mathrm{~mm}$ (4.129.4) and in women was $29.7 \mathrm{~mm}$ (19.6-47.5) (Table 1). There was a significant increase in fat superficial to the hamstring tendons in women compared with men $(P<0.001)$, despite there being no significant difference in BMI $(P=0.5)$. The correlation of the fat distribution and the BMI was equivalent between male and female patients with $R^{2} 0.36$ and 0.33 , respectively (Figure 1).

\section{Discussion}

This study supports our interesting observations in the fact that there is significantly more fat superficial to the pes anserinus insertion in women than in men. The hamstring tendons in this region are used for graft harvesting for knee ligament reconstructions, and this information may be useful for surgeons performing hamstring tendon grafts.

There have been studies that have previously demonstrated anatomic differences in the shape and size of male knees compared with female knees. Female knees have been noted to have a less prominent anterior condyle, an increased $Q$ angle, and a reduced medial-lateral:anterior-posterior aspect ratio [5].

Women and men differ in the proportion of body fat they have and in how that fat is distributed $[6,7]$. The difference in fat patterning is apparent even prepubertally, with girls having more peripheral fat than boys, and the magnitude of the sex difference is amplified with maturation [8]. Males amass significantly more visceral fat, whilst females predominantly accumulate subcutaneous fat [9]. As BMI increases, however, the amount of visceral fat in women increases and men have larger amounts of subcutaneous fat on their legs [6]. In our study we found that for both male and female patients as the BMI increased there was a reasonable correlation in the increase of subcutaneous fat distribution overlying the hamstring tendons. The amount of increased subcutaneous fat was independent of BMI and purely related to gender.

The clinical application of this study relates to surgical exposure of the hamstrings tendons. Preoperative planning in female patients may require deeper retractors to be available, or a change in the orientation and size of the incision to ensure that adequate exposure is gained with increased fat superficial to the tendons.

Our study has limitations. The use of BMI as a correction to standardise the different groups introduces potential bias. It is an index, not a direct measure, and can be influenced by a variety of factors, such as ethnicity, that were not accounted for. The MRI scans performed in women were of premenopausal women only. The fat distribution of postmenopausal women is significantly different from that of premenopausal women and approaches, but never equals, 
that of men [7]. However surgery requiring use of hamstring tendons for reconstruction is rarely performed on postmenopausal women. Physical conditioning has been shown to be effective in both young and middle aged women in reducing subcutaneous fat [10], and our study MRI scans were performed in patients for assessment of patellar instability. If our study had been performed on an elite athlete population our results may have differed. The male and female patient groups had similar activity levels, and there were no elite athletes in our study population.

\section{Conclusions}

Our evaluation of a prospective series of MRI scans has shown that there is significantly more fat superficial to the insertion of the hamstring tendons in women than in men. This effect is independent of BMI and may influence exposure during hamstring tendon graft harvesting.

\section{Disclaimer}

No author, their immediate family, or any research foundation with which they are affiliated has received any financial payments or other benefits from any commercial entity related to the subject of this paper.

\section{Conflict of Interests}

The authors declare that there is no conflict of interests regarding the publication of this paper.

\section{Acknowledgments}

The authors would like to thank Mr. Jonathan Eldridge for the use of his patients within the study.

\section{References}

[1] D. Gallagher, M. Visser, D. Sepúlveda, R. N. Pierson, T. Harris, and S. B. Heymsfieid, "How useful is body mass index for comparison of body fatness across age, sex, and ethnic groups?" American Journal of Epidemiology, vol. 143, no. 3, pp. 228-239, 1996.

[2] T. K. Fehring, S. M. Odum, J. Hughes, B. D. Springer, and W. B. Beaver Jr., "Differences between the sexes in the anatomy of the anterior condyle of the knee," Journal of Bone and Joint Surgery A, vol. 91, no. 10, pp. 2335-2341, 2009.

[3] K. M. Varadarajan, T. J. Gill, A. A. Freiberg, H. E. Rubash, and G. Li, "Gender differences in trochlear groove orientation and rotational kinematics of human knees," Journal of Orthopaedic Research, vol. 27, no. 7, pp. 871-878, 2009.

[4] N. R. Howells, A. J. Barnett, N. Ahearn, A. Ansari, and J. D. Eldridge, "Medial patellofemoral ligament reconstruction: a prospective outcome assssment of a large single centre series," The Journal of Bone and Joint Surgery (British), vol. 94, no. 9, pp. 1202-1208, 2012.

[5] S. Conley, A. Rosenberg, and R. Crowninshield, "The female knee: anatomic variations," Journal of the American Academy of Orthopaedic Surgeons, vol. 15, no. 1, pp. S31-S36, 2007.
[6] M. L. Power and J. Schulkin, "Sex differences in fat storage, fat metabolism, and the health risks from obesity: possible evolutionary origins," British Journal of Nutrition, vol. 99, no. 5, pp. 931-940, 2008.

[7] C. J. Ley, B. Lees, and J. C. Stevenson, "Sex- and menopauseassociated changes in body-fat distribution," American Journal of Clinical Nutrition, vol. 55, no. 5, pp. 950-954, 1992.

[8] R. W. Taylor, A. M. Grant, S. M. Williams, and A. Goulding, "Sex differences in regional body fat distribution from pre-to postpuberty," Obesity, vol. 18, no. 7, pp. 1410-1416, 2010.

[9] E. Fuente-Martín, P. Argente-Arizón, P. Ros, J. Argente, and J. A. Chowen, "Sex differences in adipose tissue: it is not only a question of quantity and distribution," Adipocyte, vol. 2, pp.128134, 2013

[10] T. Abe, T. Sakurai, J. Kurata, Y. Kawakami, and T. Fukunaga, "Subcutaneous and visceral fat distribution and daily physical activity: comparison between young and middle aged women," British Journal of Sports Medicine, vol. 30, no. 4, pp. 297-300, 1996. 


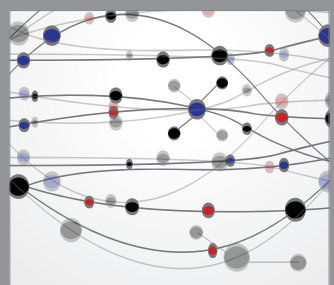

The Scientific World Journal
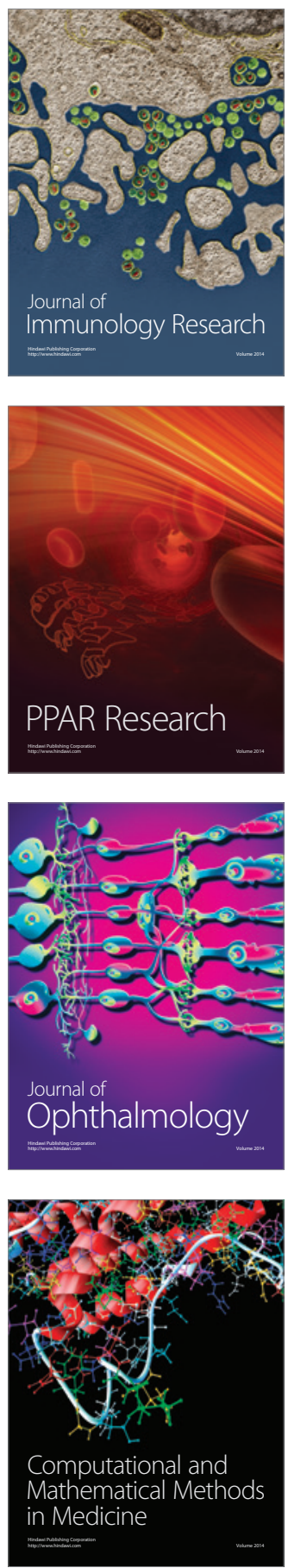

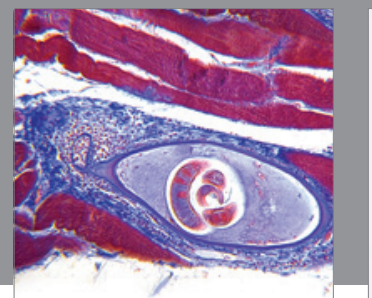

Gastroenterology

Research and Practice
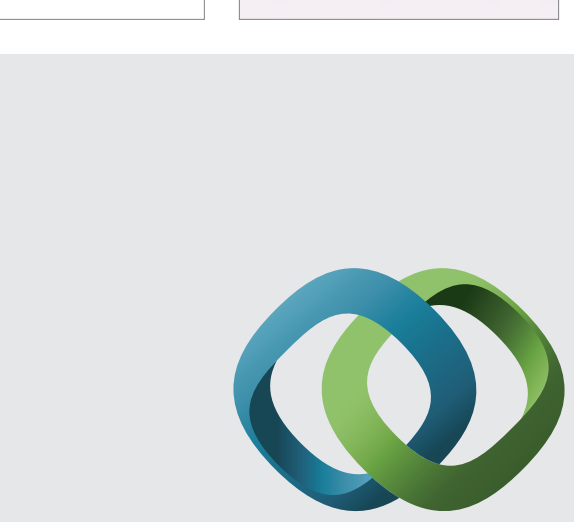

\section{Hindawi}

Submit your manuscripts at

http://www.hindawi.com
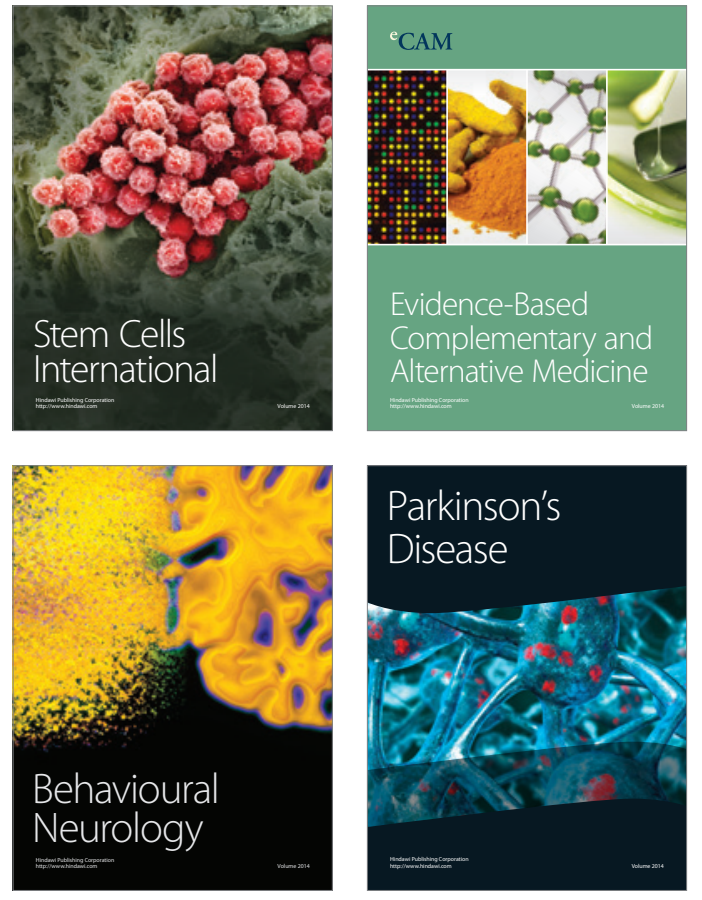
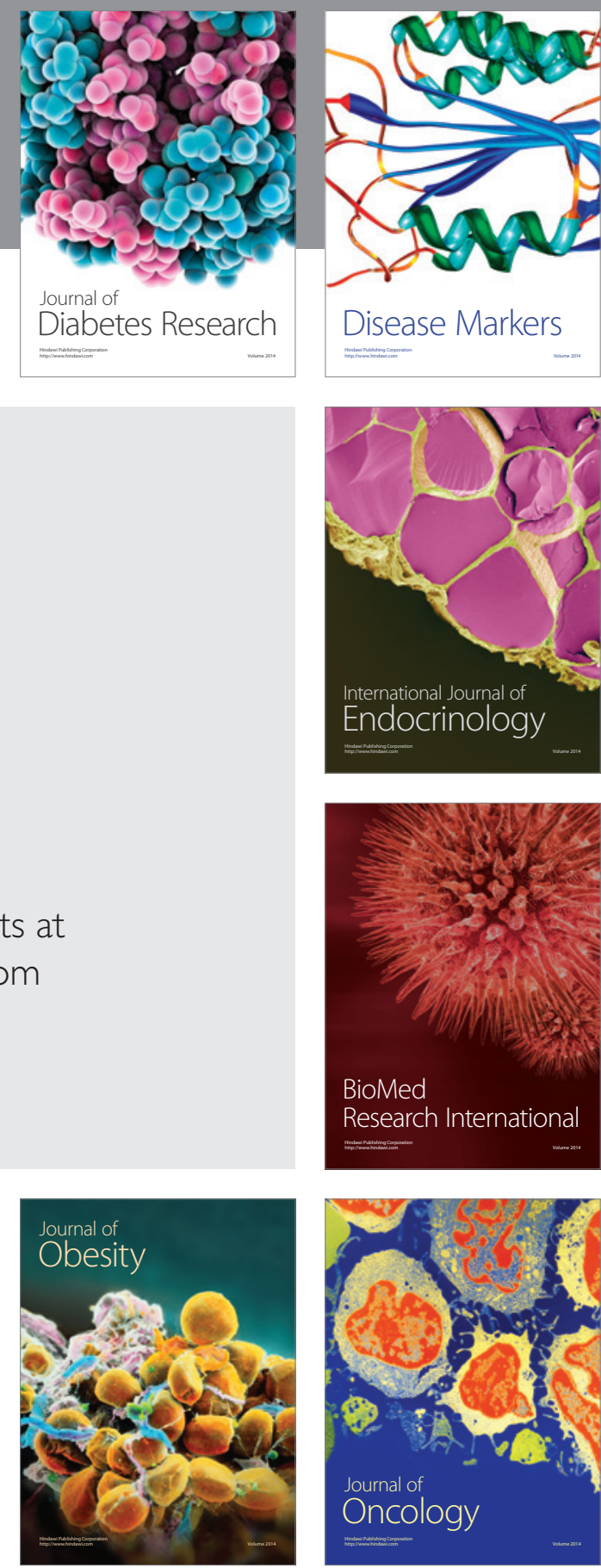

Disease Markers
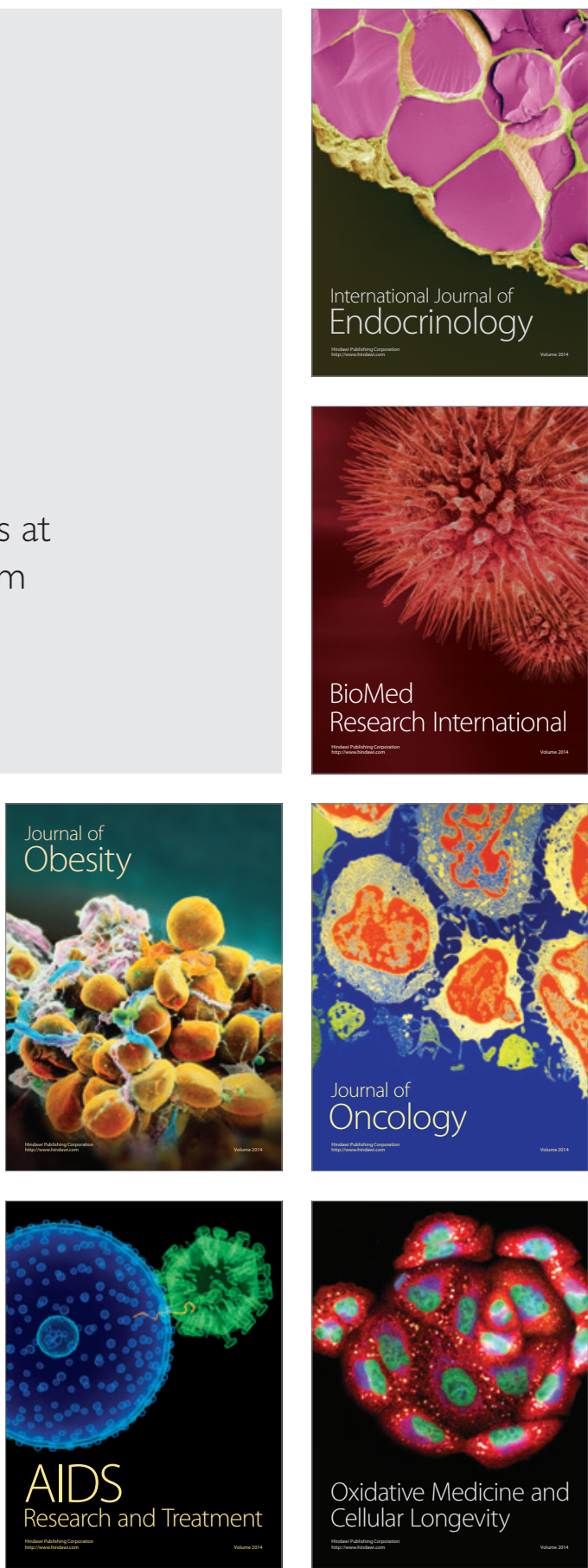